\title{
The use of ground glass in red glazes: structural 3D imaging and mechanical behaviour using optical coherence tomography and nanoindentation
}

\author{
Mitra Almasian ${ }^{1,2^{*}+}$ (1) , Mathilde Tiennot ${ }^{1 \dagger}$, Lionel D. Fiske ${ }^{2,3}$ and Erma Hermens ${ }^{1,4}$
}

\begin{abstract}
In this study we investigate the impact of the addition of colourless glass particles to red glazes, as seen in many 15th-17th-century easel paintings. With the use of reconstructions, we examined the influence of the paint preparation process on the morphological and mechanical properties of the paint film. Three sets of reconstructions were made, a control without ground glass, reconstructions with coarse or fine ground glass mixed in, and reconstructions where fine ground glass was ground jointly with the pigment oil mixture. The latter gave the desired consistency and colour based on visual inspection. The dried reconstructions were non-invasively imaged using optical coherence tomography (OCT). A data-analysis pipeline was developed for both the segmentation of the OCT images and the measurement of the size and spatial distributions of the glass particles within the glaze layer. Moreover, we used a nanoindentation protocol to measure the viscoelastic properties of the dried red glaze film. The OCT results show an expected decrease in median particle size with longer grinding-time, for which the additional grinding with pigment/oil resulted in a more narrow size distribution and a homogenous spatial distribution of the glass particles. The nanoindentation results indicate that the addition of glass particles increases the elastic and viscous moduli of the red glaze layers. The homogeneous size distribution, obtained by grinding the oil, pigment, and glass together, induces higher elastic and viscous moduli. Our imaging and analyses approach, combining OCT and nanoindentation, provides a non-invasive and quantitative investigation of glass particles in (semi-) transparent paint layers, and their effect on the mechanical properties of the glaze. The results of this study contribute to a better understanding of the artists' addition of ground glass in paint layers.
\end{abstract}

Keywords: Glass in paint, Historical recipes, Optical coherence tomography (OCT), Nanoindentation, Quantitative data- analysis, Technical art history

\section{Introduction and research aim}

The presence of colourless glass particles in paints in European easel paintings from the 15th to the 17th century, especially in transparent oil-bound red glazes, is

\footnotetext{
*Correspondence: m.almasian@amsterdamumc.nl

${ }^{\dagger} \mathrm{M}$. Almasian and M. Tiennot contributed equally to this study.

${ }^{1}$ Conservation and Science Department, Rijksmuseum Ateliergebouw,

Hobbemastraat 22, 1071 ZC Amsterdam, Netherlands

Full list of author information is available at the end of the article
}

a regular occurrence. Contemporary art technological sources recommend this practice mainly to speed up the drying process. Although adding dryers to oil paint is indeed quite common, the function of the colourless ground glass is not yet fully understood. Dietz [1] studied the extensive use of ground glass in works by Hans Holbein the Elder (c.1460/70-1524), where glass particles are mixed with a large variety of pigments in almost all paint layers, not just red glazes, and are also present in mordant layers for gilding. Spring [2] identified powdered
Springer Open

(c) The Author(s) 2021. This article is licensed under a Creative Commons Attribution 4.0 International License, which permits use, sharing, adaptation, distribution and reproduction in any medium or format, as long as you give appropriate credit to the original author(s) and the source, provide a link to the Creative Commons licence, and indicate if changes were made. The images or other third party material in this article are included in the article's Creative Commons licence, unless indicated otherwise in a credit line to the material. If material is not included in the article's Creative Commons licence and your intended use is not permitted by statutory regulation or exceeds the permitted use, you will need to obtain permission directly from the copyright holder. To view a copy of this licence, visit http://creativeco mmons.org/licenses/by/4.0/. The Creative Commons Public Domain Dedication waiver (http://creativecommons.org/publicdomain/ zero/1.0/) applies to the data made available in this article, unless otherwise stated in a credit line to the data. 
colourless glass in 15th and 16th-century German and Netherlandish school paintings in the collection of the National Gallery, London, amongst others in Jan van Eyck's Arnolfini Portrait and Annunciation, and quantified their chemical composition using SEM-EDX. Seccaroni et al. [3] proposed that the addition of ground glass was ubiquitous in Florentine paintings from 1490 to 1530 . However, Spring also established extensive use of glass in 15th and 16th-century Italian paintings from Bologna and Ferrara, and in works by Raphael $[4,5]$. In the latter it occurs mixed with various pigments and in different layers in the paint stratigraphy. Here the glass particles contain manganese, added by Italian glass makers from the late thirteenth century onwards to counteract the greenish tinge caused by iron impurities in the sand or pebbles used. Amadori [6] found a lead tin yellow in a glassy matrix as well as colourless glass particles in works by the Venetian painter Lorenzo Lotto (14801556), both materials could plausibly be connected to the local glass industry [7]. Recent research by Melo et al. [8] demonstrates the presence of glass particles, probably from a locally made glass, in two altarpieces by the Portuguese painter Francisco Joao (1563-1595). Based on the exclusive use in red glaze layers, they suggest the glass was added both for its 'assumed' siccative properties, which they connect to the effect of the lower amount of oil in the paint as the glass adds bulk, as well as for its transparency, making it especially suitable as an extender for glazes.

The siccative effect of ground glass is indeed often referred to in historical recipes [9]. For example, the Tegernsee manuscript [10], c. 1500, advised adding ground venetian glass to red lake paints to improve their drying time. The much later De Mayerne Manuscript [11], c. 1620-1655, in a description of paints that have little or no body (covering strength), lists ground glass amongst other well-known dryers, stating that: '...colours that do not dry well, are helped if verdigris is added, or white vitriol, or crystal glass powdered to being impalpable, or calcined and quenched in cold water and then dried and crushed to a very fine powder.' Marshall Smith, in his treatise The Art of Painting... [1692] [12], emphasises the fact that the ground glass has no impact on the colours, and refers to Italy for this practice, probably indicating with 'the whitest glass' the pure Italian cristallo: '... take the whitest glass, beat it very fine in a Mortar, and grind it in water to an impalpable powder; being thoroughly dry, it will dry all Colours without drying Oyle, and not in the least Tinge the purest Colours, as White, Ultramarine, \&c. and is much used in Italy' Glass is hard to grind and, as both Mayerne and Smith state, needs to be so fine that it is 'impalpable'. When not ground fine enough the glass particles will remain prominently visible. The fineness required is difficult to estimate as the recipes provide only qualitative descriptions. For example, an indication is given in the late 16th-century French BnF. 640 manuscript, which recommends that the cristallin glass: '... because it would be too difficult to grind by itself, one must redden it on the fire, then when entirely red throw it into cold water, \& it will crumble \& pulverize easily for grinding it afterward. Being well ground with a lot of water, it resembles ground lead white, but for all this it has no body...' [13, fol.57r]. 'Impalpable' is a sensory marker of touch, while resembling lead white is a visual effect which, indeed occurs quite quickly when grinding glass into fine particles. The reference to 'no body' most likely indicates its translucency in a liquid, such as oil, if indeed ground very finely. This is mentioned in another note in BnF 640 (fol.58r) which states that in oil, cristallin has no body. Lutzenberger et al. [9] found glass particles in German and Netherlandish paintings measuring between 1 and 15 microns, and up till 10 microns in red lake glazes. In Italian paintings the particle size could range from 2 to 50 microns. Spring [2] found that the glass particles in Netherlandish and German paintings averaged 2-5 microns, and measured up to 50 microns in Italian paintings. Melo et al. found a wide range of glass particles mostly from c. 2 microns up to 30 microns, with a rare very large 55 microns particle [8].

Historical recipes are also rather ambiguous on how and when to add the powdered glass to the paint and how to incorporate it. The Portuguese painter Felipe Nunes, in De Arte de la Pintura (1615), explicitly states to never grind the glass together with the paint mixture on the grinding stone, 'so that the paint is kept fresh for later use', probably warning against the effect of accelerated drying [14]. However, the mid 16th-century Padua manuscript recommends to '...grind them [lake, indigo, lamp black] with oil then take glass ground to a fine powder, and incorporate with the colours by grinding them together again' [15]. One other functional use of ground glass is to make the grinding of certain pigments, such as orpiment, easier, which could also have been the case with other pigments in glass containing layers [9].

Based on observations during reconstructions of oilbound red lake glazes, Lutzenberger et al. [9] mention the 'physico-optical considerations or to increase colour depth', which, they state, are not mentioned in the treatises but due to the frequent occurrence of glass in glazes seem plausible. In the BnF 640 manuscript, under the heading 'glazing', powdered glass is recommended as an addition for colours other than lakes and verdigris. One should mix in calcined and ground cristallo '... which also has no body \& lightens the density of the others [colours]..., seemingly connecting the addition of glass with less body again, i.e. increased transparency and 
less covering power. [13, fol.65v]. Physico-optical aspects could also be indicated here although this would need the glass to be very finely ground, as we will elaborate on in our reconstructions.

From the studies mentioned above [2, 7-9] it seems that painters used locally available glass. For example, Lutzenberger et al. [9] found mostly soda ash glass in Italian paintings, and potassium rich glass in German an Netherlandish paintings. This may have implications for the use of glass as a siccative. However, in this study we will not investigate the drying properties or the chemical composition of the added glass. Instead, we will focus on both the size and spatial distribution of the glass particles, and their respective impact on the mechanical properties of the paint. Understanding the morphology of the glass particles and their behaviour in reconstructed paints will be a first step towards understand their influence on the optical properties of the glaze. Such measurements of morphological properties can be used in calculations and models to predict optical and mechanical behaviour. Although ground glass is not only found in red glaze layers but, as said, also in other paint, mordant and ground layers, in this paper we will focus on red lake glaze recipes only. We will use reconstructions with one type of (modern) soda glass, preparing the glass as described in the recipes and as seen from the range of particle sizes found in original layers $[2,8,9]$, by hand grinding it, and adding it to or grinding it together with the pigment-oil mixture.

The purpose of this study was to investigate the effect of different preparation processes (used for the addition of ground glass) on the morphology and mechanical properties of the red lake glaze reconstructions. Our aim was to study these properties with a microscopic resolution and find quantitative expressions. Therefore, optical coherence tomography (OCT) was used to noninvasively visualize the glass particles in the reconstructions. In semi-transparent layers, OCT can be a powerful imaging tool to visualize structures and inclusions below the surface, such as glass particles in our case. A custom written data-analysis pipe-line was used to extract size and spatial distributions of the ground glass particles from the 3D OCT images. In recent years, OCT has been more widely applied to visualize paint layers [16-18] including paint layers containing smalt [19]. Here, we extend our analysis to obtain quantitative parameters from OCT images. Furthermore, we have investigated the viscoelastic behaviour of red glaze paints using a nanoindentation protocol [20], to quantify the influence of the addition of glass and of the grinding process on the elastic and viscous moduli of the dried glaze, as alluded to in the recipes.

\section{Reconstructions}

A mixture of $4.5 \mathrm{~g}$ of heat bodied linseed oil, cold-pressed from organic linseeds and boiled for $6.5 \mathrm{~h}$, plus $3.5 \mathrm{~g}$ of cochineal red lake pigment was used for the reference reconstructions (B1).

To obtain a better understanding of what exactly happens in both processes: adding the ground glass to a ready-made paint and just mixing it in with a palette knife, or grinding the glass, pigment and binding medium together, we decided to reconstruct both processes. We used a soda glass which was blown into a very thin bubble making breaking and grinding easier. The glass was hand ground in a pestle and mortar for 5 min which did make it look like an opaque white powder, similar to lead white as mentioned in the BnF640 recipe, and for $10 \mathrm{~min}$, which increased its fineness considerably.

To prepare sample (B2), $1 \mathrm{~g}$ of ground glass was added to the initial red lake/oil mixture (B1) and mixed in with a palette knife, which, as could be expected, showed a gritty texture with very visible coarse glass particles. The second mixture (B3), made with longer ground (10 min) and hence very fine glass particles, still showed the presence of the ground glass prominently. For (B4) oil, pigment, and glass particles were further ground together at different lengths of time (Fig. 1).

So far, we found no indications in the recipes that quantify grinding times, and hence the optimal grinding

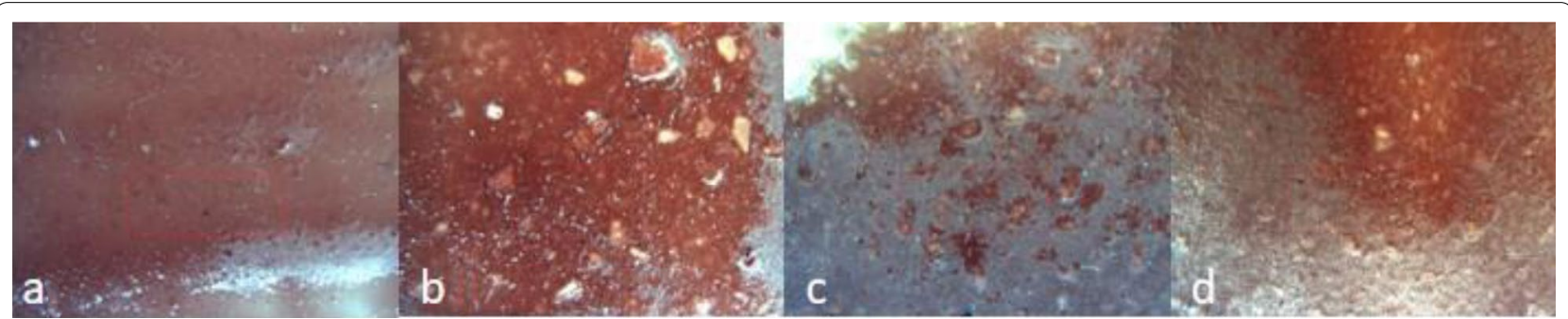

Fig. 1 Images of the reconstructions collected with the integrated camera of the OCT device. From left to right: a no glass, $\mathbf{b}$ added coarse glass, $\mathbf{c}$ added fine glass, $\mathbf{d}$ ground together for $>25 \mathrm{~min}$ 
time for (B4) was determined through experiments, where after 25 min of additional grinding together with the oil/pigment mixture, the rather loud sound of the grinding reduced substantially and the paint became very smooth. We accepted this as a sensory marker indicating the needed amount of grinding. We also noted that this paint (B4) was easier to apply than (B1), and achieved a deep saturation of tone while remaining very transparent upon visual inspection and leaving no glass particles visible to the naked eye.

All the mixtures were applied with a brush on a panel prepared with a chalk and animal skin glue ground (10\%) covered with an unpigmented isolation layer of heat bodied linseed oil which would prevent the oil from the paint to be absorbed by the chalk ground. This application resulted in very slight differences in thickness of the applied red glaze layers, but stayed close to contemporary workshop practice. The reconstructions dried naturally for two years.

\section{Scientific methodologies}

\section{Morphological imaging using optical coherence}

\section{tomography}

Volumetric OCT images were collected using a Thorlabs Telesto polarization sensitive OCT system, with a centre wavelength at $1300 \mathrm{~nm}$, and an axial and lateral resolution of 5.5 and $13.0 \mu \mathrm{m}$ in air, respectively. 3D images of $(x, y, z) 5.00$ by 2.00 by $1.57 \mathrm{~mm}$ with 1024 by 1024 by 450 pixels were collected from each sample. The focal point of the sample arm was set at $0.5 \mathrm{~mm}$ from the zerodelay position.

The refractive index of the oil/pigment mixture at $1300 \mathrm{~nm}$ was experimentally determined, from additionally collected OCT images, to be 1.56 . This value was used to convert the OCT-depth axis from optical path length to physical length. The refractive index of the glass particles was estimated to be 1.6, which falls in the range of refractive indices expected for glass at $1300 \mathrm{~nm}$ [21].

A semi-automated data analysis pipeline was developed in order to process and analyse 3D OCT images. Initially, the collected images were averaged and preprocessed using ImageJ, to minimize speckle and maximize contrast. The images were averaged to 714 by 286 by 225 pixels $(x, y, z)$, despeckled and sharpened with the existing Image $J$ functionalities. Next, the layers were classified using an off the shelf random forest classifier called Ilastik (Interactive Learning and Segmentation Toolkit) [22]. This classification is probabilistic, and the program outputs a series of $3 \mathrm{D}$ images whose pixel values are the probability of being glass, the oil/pigment medium, or the boundary of the glaze layer. These probability maps were then read into Matlab 2020a and then a maximum likelihood approach is used to find the approximate boundary locations. Due to the presence of speckle, noise, and various imaging artifacts the boundary segmentation can contain errors such as discontinuities. To account for this, the locations of the boundaries were smoothed, and physical ordering of the layers is enforced where needed. After this processing, the index of refraction of the layers is accounted for and the specific layer geometry is measured. The layer shapes can be clearly seen in Fig. 7.

The segmentation of the glass particles was performed fully in Ilastik and no additional conditions or smoothing was applied. The objects were identified and segmented and a binary file was then read into Matlab 2020a containing all the identified pixel locations to calculate the volume of the glass particles. To remove artifacts, these images were filtered to exclude volumes smaller than 20 pixels. The threshold of 20 pixels was determined to be reasonable by visual inspection. Images of the segmentation and the original OCT images were overlaid and found to be in good agreement based on visual inspection. An example of the segmented particles can be seen in Fig. 4. From these filtered images, the size distribution, spatial distribution and volume fraction of glass particles were calculated. A complete diagram of this data processing pipeline is shown in Fig. 2.

\section{Evaluation of the mechanical behaviour using nanoindentation}

The addition of glass to the red glazes may have an impact on their mechanical behaviour. The grinding process and the resulting size and distribution of the particles may also influence the macroscopic behaviour of the paint layer. To evaluate the elastic and viscous properties of the dried reconstructions at microscale, we used a specifically designed nanoindentation protocol, based on a depth-controlled analysis [20]. For this protocol, a borosilicate sphere measuring up to $70 \mu \mathrm{m}$ in diameter, is glued on top of a micromachined glass cantilever. An optical fibre attached to this specially designed ferruletop probe monitors the bending of the device while in contact with the sample (Fig. 3). A camera placed on top of the equipment allows the proper localisation of the measurement on the surface.

After an approach phase at a $5 \mu \mathrm{m} . \mathrm{s}^{-1}$ speed, a $2 \mathrm{~s}$ loading reaches the indentation depth $\mathrm{d}_{\mathrm{i}}=2 \mu \mathrm{m}$, where a relaxation time of $20 \mathrm{~s}$ is performed. Dynamic Mechanical Analyses (DMA) via small oscillations at an $\mathrm{A}=0.05 \mu \mathrm{m}$ amplitude around the indentation depth during a frequency sweep of five frequencies ranging 


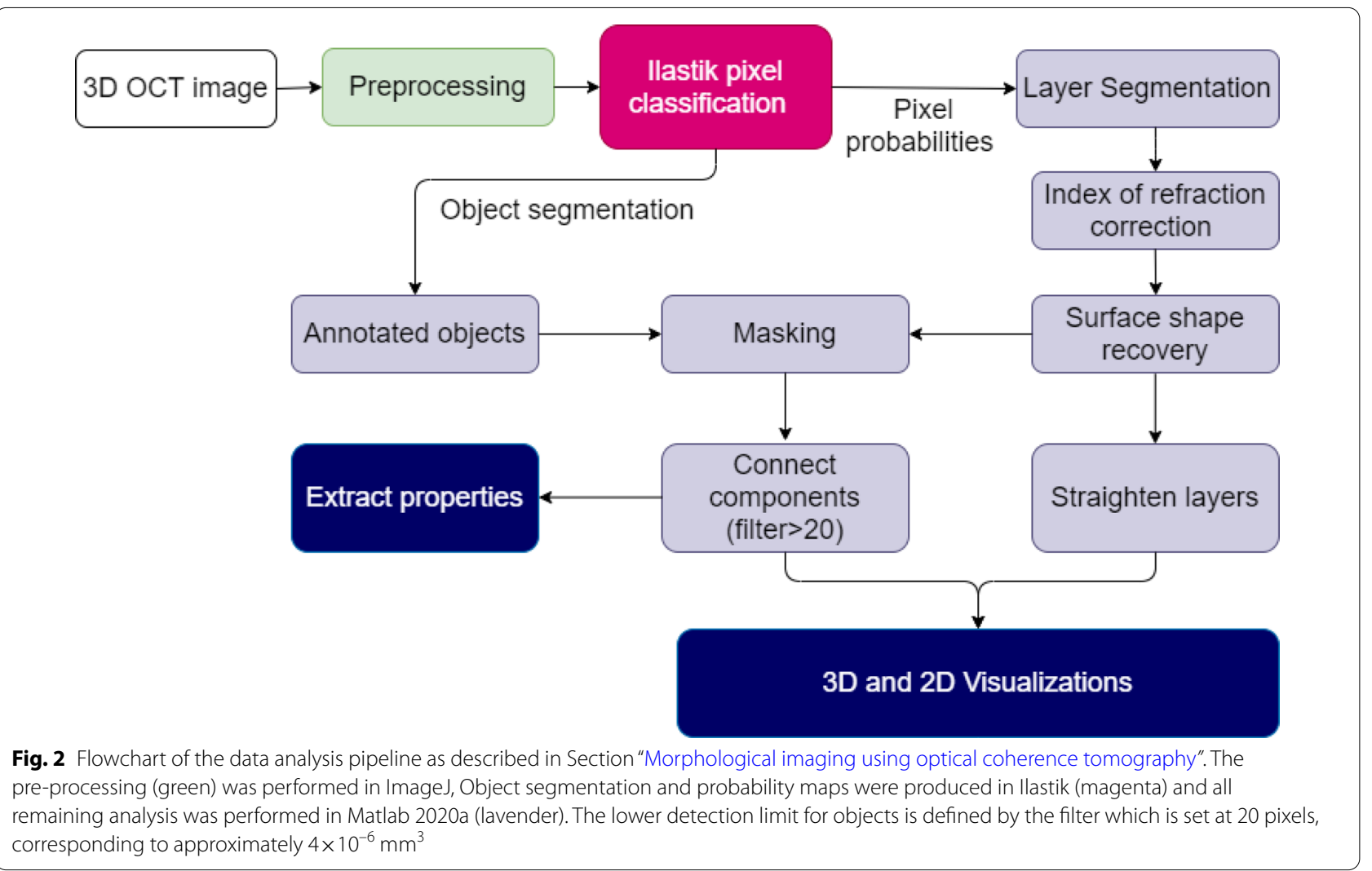

from $f_{1}=1 \mathrm{~Hz}$ to $f_{5}=10 \mathrm{~Hz}$, allow the evaluation at each frequency of the storage E' and loss E" moduli of the material. The storage modulus represents the ability of the material to store energy and relates to the elastic part of the behaviour, and the loss modulus represents the part of the energy dissipated and underlines the viscous properties of the material. These properties characterize the viscoelasticity of the glaze after natural drying.

For this paper, we focused on the properties of samples B1 (no glass), B2 (coarse glass) and B4 (ground together for $\mathrm{t}>25 \mathrm{~min}$ ), measured using this indentation protocol. The aim of these indentation measurements is to evaluate the mechanical properties of the dried paint film and their modification when glass particles are added, and to specifically study the influence of the grinding process on the elastic and viscous behaviour of the oil/pigment mixture. Therefore, we performed the indentation measurements on the glaze surface, and avoided the glass particles by visual inspection.

\section{Results}

\section{D visualisation by $O C T$}

OCT volumetric images were successfully collected from each sample, clearly visualizing the glass particles. Using custom-developed software the glass particles were

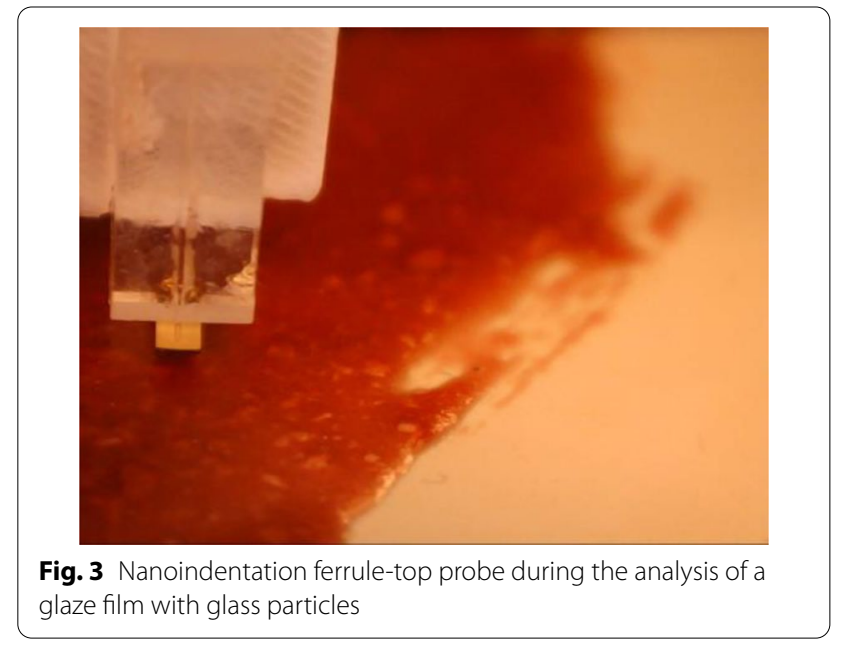

segmented from these 3D images (Fig. 4). Figure 5 shows camera images with the selected region of interest (ROI) for OCT imaging together with an OCT cross-section. The segmented dataset is subsequently used to calculate morphological properties of the reconstructions. Table 1 summarizes the obtained properties: layer thickness, total number of glass particles, total glass volume, ratio of glass to oil/pigment matrix expressed in volume percentage, and median particle size expressed in $\mathrm{mm}^{3}$. 


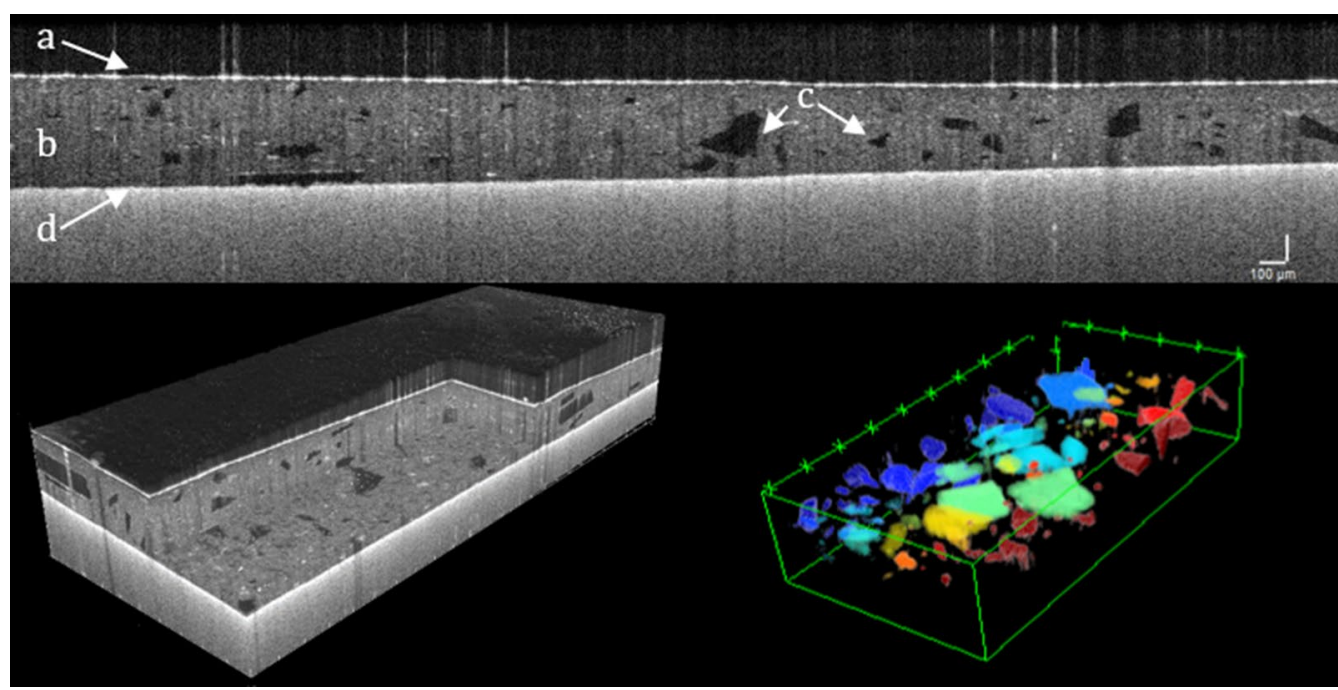

Fig. 4 OCT cross-sectional image a air glaze transition, b glaze, c glass particles, $\mathbf{d}$ glass support transition. OCT volumetric image. Segmented and colour-labelled glass particle interior of the glaze. The OCT images at $1300 \mathrm{~nm}$ do not provide enough contrast between the oil matrix and pigment particles, which are not visible in the OCT images

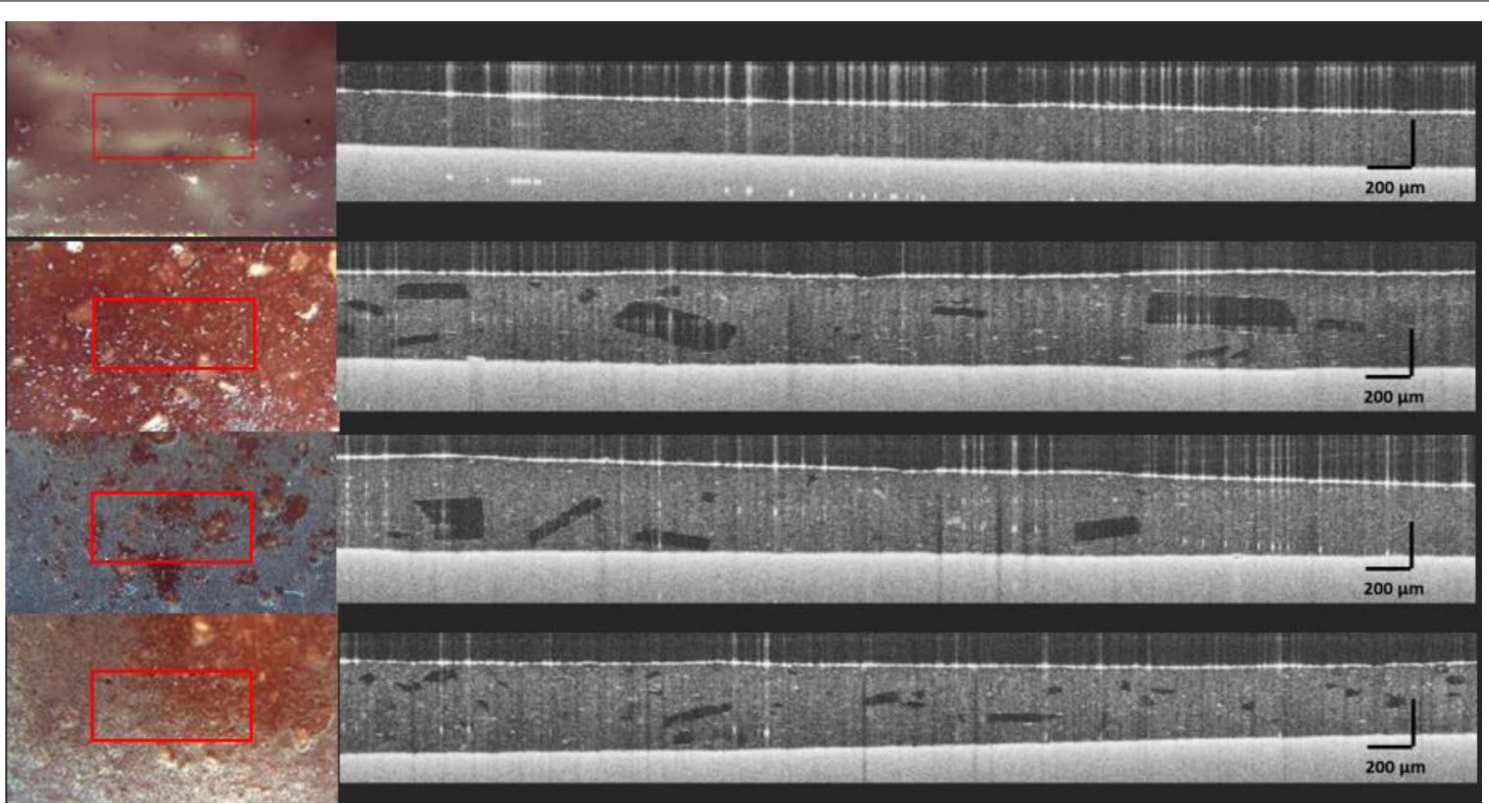

Fig. 5 Region of interest (ROI) of volumetric OCT scans and OCT cross sectional images of the reconstructions. From top to bottom: B1 (no glass), B2 (coarse glass added), B3 (fine glass added) and B4 (ground together with glass for $>25 \mathrm{~min}$ )

\section{Size distribution of glass particles}

The size distribution of glass particles for samples B2-4, obtained by OCT, are plotted in histograms in Fig. 6 . The median particle size follows the expected trend; decreasing with an increase in grind-time. The percentage of glass in the total glaze volume was calculated to correct for the slight thickness differences between the three reconstructions. Here, the glass volume percentage of sample B3 deviates form B2 and B4 while the paint mixture contained the same amount of glass. This can be rationalized by the inhomogeneous distribution of glass particles in the glaze and the presence of large particles within the imaged volume. A distinct narrowing of the size distribution is visible for the sample where the glass 
Table 1 Mean thickness and properties of the glass particles (total number and volume, median size) for the four samples, measured by our OCT protocol

\begin{tabular}{lllllll}
\hline$\#$ & Glass content & Mean, std thickness layer & $\begin{array}{l}\text { Total } \\
\text { particle } \\
\text { number }\end{array}$ & Total glass volume & Volume\% glass & Median particle size \\
\hline B1 & No glass & $0.22 \pm 0.01 \mathrm{~mm}$ & - & - & - & - \\
B2 & Coarse glass & $0.37 \pm 0.03 \mathrm{~mm}$ & 206 & $0.1 \mathrm{~mm}^{3}$ & $3.2 \%$ & $3.5 \times 10^{-5} \mathrm{~mm}^{3}$ \\
B3 & Fine glass & $0.40 \pm 0.02 \mathrm{~mm}$ & 313 & $0.3 \mathrm{~mm}^{3}$ & $4.7 \%$ & $2.5 \times 10^{-5} \mathrm{~mm}^{3}$ \\
B4 & $\begin{array}{l}\text { Fine glass added to paint and } \\
\text { ground together for 25 min }\end{array}$ & $0.36 \pm 0.04 \mathrm{~mm}$ & 632 & $0.1 \mathrm{~mm}^{3}$ & $2.4 \%$ & $2.3 \times 10^{-5} \mathrm{~mm}^{3}$ \\
\hline
\end{tabular}

is ground together with the oil/pigment mixture (sample B4).

\section{Spatial distribution of glass particles}

A scatterplot depicting the centre position of the glass particles in reference to the top and bottom of the glaze layers is shown in Fig. 7. The size of the spheres is proportional to the volume of the glass particles. In all three samples (B2-B4) the glass particles are distributed at similar distances from the top and bottom of the layer, showing no evidence of sinking to the bottom. Clearly, sample (B4), where glass is ground together with the oil/ pigment mixture for an additional $25 \mathrm{~min}$, corresponds to a more homogeneous distribution of the glass particles.

\section{Impact of the addition of glass particles and the grinding process on the mechanical properties of the glaze}

We evaluated the elastic and viscous properties of the naturally dried red glazes, with respect to the number, volume and distribution of the glass particles added to the paint. The aim was to measure the impact of glass on the macroscopic stiffness and viscosity of the red glaze. The

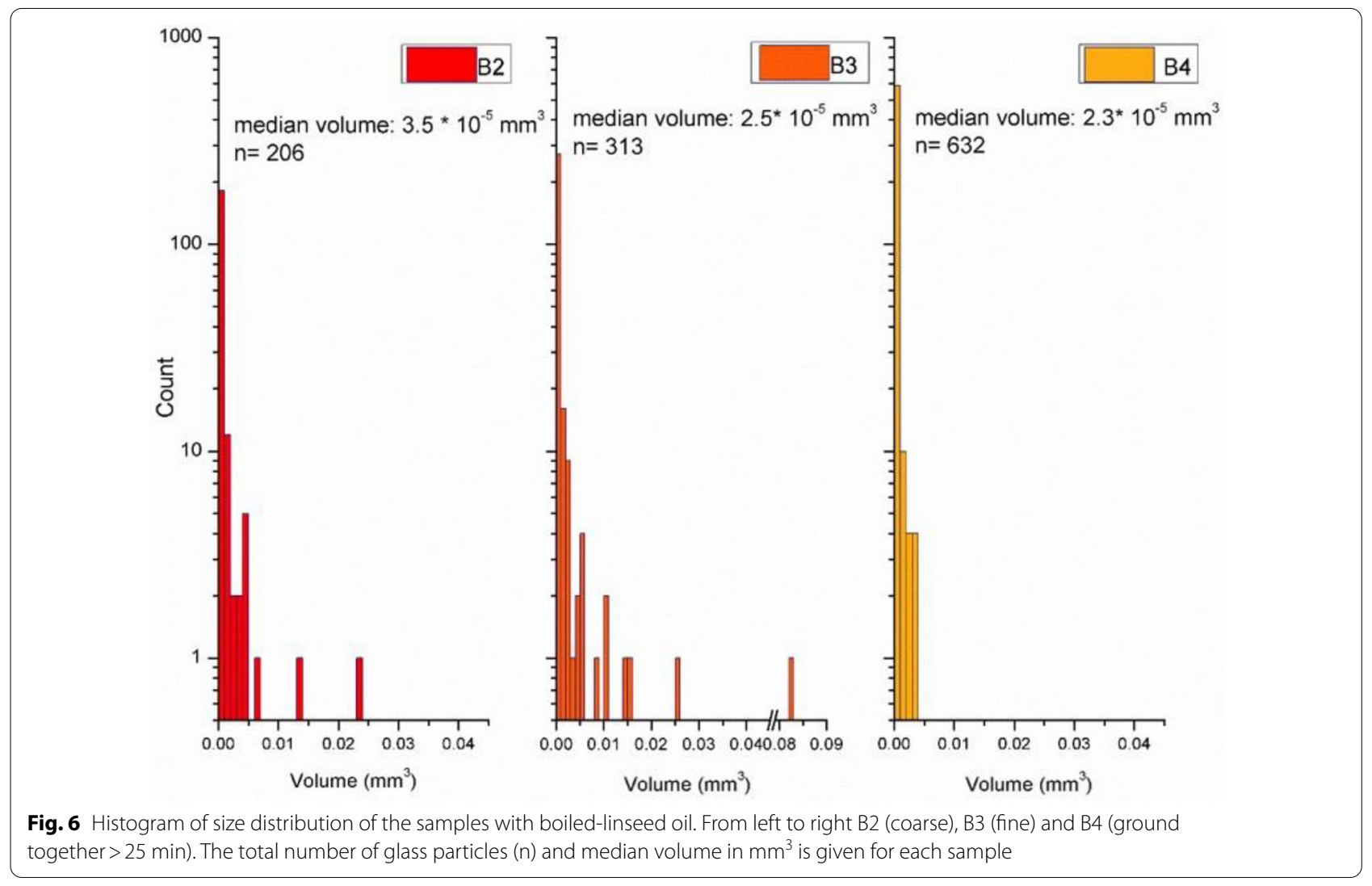




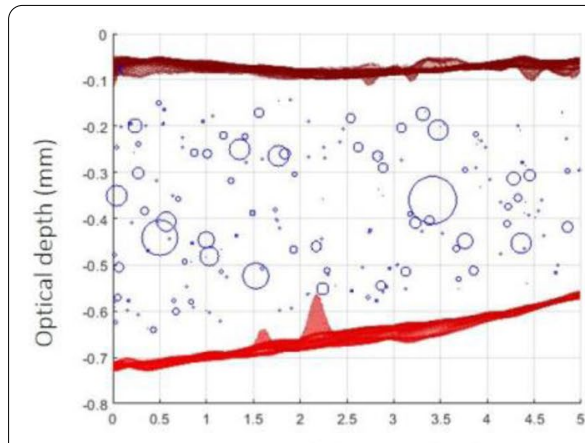

Lateral direction $(\mathrm{mm})$

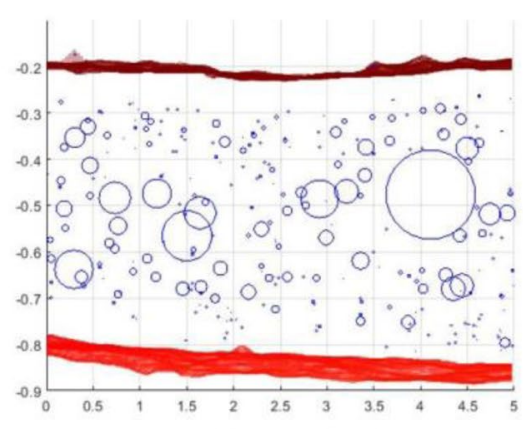

Lateral direction $(\mathrm{mm})$

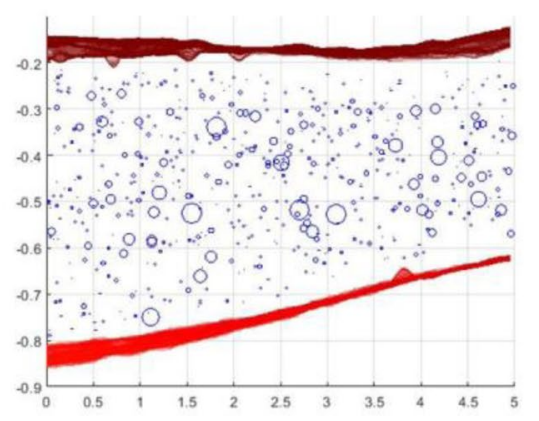

Lateral direction ( $\mathrm{mm}$ )

Fig. 7 The segmented top and bottom of the red glaze layer overlayed with scatter plots depicting the spatial distribution of the glass particles in reconstruction. From left to right: added coarse glass (B2), added fine glass (B3), ground together (B4). The scatterplots show the centre position of each glass particle, the size of the spheres is proportional to the volume of the glass particles



Fig. 8 Storage and loss moduli of the sample B1 (with no glass particles) at an indentation depth of $2 \mu \mathrm{m}$ measured for a frequency sweep of 5 measurements from 1 to $10 \mathrm{~Hz}$ at an amplitude of $A=0.05 \mu \mathrm{m}$; the increase of the moduli with the frequency indicates the viscoelastic behaviour

differences in the mechanical properties may be related to the drying processes involved in the paint films.

The storage and loss moduli of the reference glaze without glass (B1), obtained by dynamic mechanical analysis, are presented in Fig. 8. They were compared to the properties after the addition of glass. A viscoelastic behaviour is observed for the three paint films, illustrated by the increase of the moduli along the frequency sweep (Table 2).

The reference paint film (B1) shows at $\mathrm{f}_{1}$ an average storage modulus of $\mathrm{E}_{\text {ref }}^{\prime}=5.75 \mathrm{MPa}$ and a loss modulus of $\mathrm{E}^{\prime \prime}{ }_{\text {ref }}=0.74 \mathrm{MPa}$.

When glass is added, we measured respectively at the first frequency a storage and loss modulus of $\mathrm{E}_{\text {coarse }}^{\prime}$ glass $=11.2 \mathrm{MPa}$ and $\mathrm{E}^{\prime \prime}{ }_{\text {coarse glass }}=1.84 \mathrm{MPa}$ for the sample (B2) with coarse particles and $\mathrm{E}_{\text {extra ground glass }}^{\prime}=23.4 \mathrm{MPa}$ and $\mathrm{E}^{\prime \prime}$ extra ground glass $=14.5 \mathrm{MPa}$ for the sample (B4) with glass ground together with oil/pigment mixture. These properties are summarized in Table 2.

We therefore observe that the addition of glass induces an increase of the elastic and viscous moduli of the dried red glazes. Moreover, the differences in storage $E^{\prime}$ and loss E"moduli measured between the glazes with coarse particles (B2) and with particles ground together (B4) indicate that the particle size and its distribution influence the paint behaviour: the finer the glass particles and the more homogeneous the distribution of their size, the higher both moduli of the overall paint layer.

Table 2 Mean values of the storage E' and loss E" moduli measured by dynamic mechanical analysis on samples B1 (no glass) B2 (coarse glass added), and B4 (ground together)

\begin{tabular}{|c|c|c|c|c|c|c|}
\hline \multirow{2}{*}{$\begin{array}{l}\text { Moduli (MPa) } \\
\text { F (Hz) }\end{array}$} & \multicolumn{2}{|c|}{ No glass (reference) } & \multicolumn{2}{|c|}{ Coarse particles } & \multicolumn{2}{|c|}{$\begin{array}{l}\text { Glass particles extra } \\
\text { ground }\end{array}$} \\
\hline & $\mathrm{E}^{\prime}$ & $\mathrm{E}^{\prime \prime}$ & $\mathbf{E}^{\prime}$ & $\mathrm{E}^{\prime \prime}$ & $\mathbf{E}^{\prime}$ & $\mathrm{E}^{\prime \prime}$ \\
\hline 1.00 & 5.75 & 0.74 & 11.12 & 1.84 & 23.37 & 14.52 \\
\hline 1.78 & 6.00 & 0.99 & 11.90 & 2.94 & 28.00 & 19.84 \\
\hline 3.16 & 6.45 & 1.36 & 12.62 & 3.59 & 32.81 & 26.59 \\
\hline 5.62 & 6.64 & 1.87 & 13.61 & 4.78 & 39.02 & 37.83 \\
\hline 10.00 & 6.89 & 2.67 & 14.34 & 6.51 & 43.10 & 51.10 \\
\hline
\end{tabular}




\section{Discussion}

In this study, we have successfully applied OCT and nanoindentation to image and quantify morphology and mechanical properties of red lake glazes containing ground glass. We evaluated the influence of the grinding process on the size and spatial distribution of glass particles in the red glaze paint and related these to the measured mechanical properties of the reconstruction.

This approach allows us to investigate the questions on the prevalent studio practice. For how long was the glass ground? Was the ground glass added to the paint just before applying it, as was advised by Felipe Nunes [14], or was it actually ground with the prepared paint on the grinding slab, as explained in the Padua manuscript [15] and BnF 640 [13]. We should note that most recipes do not contain either information. Although the variety in the visual appearance of the reconstructed glaze paints is beyond the scope of this paper, our observations with the naked eye suggest an increase in depth of colour after glass, pigment and oil were ground together for at least $25 \mathrm{~min}$. Visualizing the morphology of the glaze and glass particles is an important step towards understanding the influence of the addition of glass on colour and visual appearance.

From the OCT results we found that an increase in grinding-time of the glass before adding it to the paint as in samples B2-3, as expected, corresponds to a decrease in median size of the glass particles together with an increase in the number of particles. Using manual grinding methods, as would have been done in the 15th17th- century workshop, it was difficult to decrease the particle size further or achieve a very homogenous size distribution. However, a much narrower size distribution was observed for sample (B4), for which the glass and oil/ pigment mixture were ground together for an additional $25 \mathrm{~min}$. As a result of the narrower size distribution, the glass particles are more homogeneously distributed in the oil/pigment mixture in this sample compared to the other samples. Although not part of our analysis, it may be assumed that the grinding together of the oil-pigmentglass mixture will also have an impact on the size distribution of the red lake particles. As mentioned in the introduction, glass was sometimes added to facilitate the grinding process of certain pigments [9].

The accuracy for detecting glass particles using OCT is determined by the imaging contrast, resolution and analysis. In the described analysis pipeline, the lower detection limit is 20 pixels, corresponding to approximately $4 \times 10^{-6} \mathrm{~mm}^{3}$. Therefore, below this limit, glass particles were not detected. As this limit is approximately ten times smaller than the median particles size, we assume that it will not hamper our results significantly. The OCT results show similar amounts of total glass volume
(Table 1) for sample (B2), (B3) and (B4). This is in agreement with the sample preparation, in which the same amounts (in weight) of oil, pigment and glass were used. The deviation of sample B3, can be attributed to the presence of large glass particles within the imaged ROI. Furthermore, we note that the cochineal particles were not distinguished on the OCT images.

Based on the OCT observations and the equal total glass volume in sample (B2) and (B4), the impact of the addition of glass (B2) and the differences induced by the grinding process (B4) on the mechanical behaviour of the dried paint film can be discussed using the nanoindentation results. The presented nanoindentation experiments performed at a $2 \mu \mathrm{m}$ depth on the surface indicate that the addition of glass particles leads to a higher stiffness as well as a higher viscous modulus of the red glaze. For the same volume of material, the $>25$ min grinding process of the oil-pigment-glass mixture (B4) doubles the number of glass particles (Table 1), which have a smaller median particle size (Fig. 6). A decrease in the size of the particles is related to an increase of their surface area, and hence improves interaction with the oil-pigment mixture. A similar volume of glass combined with a narrow size distribution of the particles-as in sample (B4) - significantly modifies the viscoelasticity of the layer. Thus, the grinding process influences the size distribution of the glass particles, and has a direct repercussion on the macroscopic mechanical behaviour of the red glaze after drying for two years.

With the presented work we aim to contribute to the quantitative study of cultural heritage materials. The OCT data-analysis pipeline allows for segmentation of layers and inclusions such as glass particles, from which size and spatial distribution can be obtained, providing the opportunity to compare morphological differences between samples. Furthermore, semi-automated analysis of the data can replace time consuming manual segmentation as well as minimize user-introduced biases [23]. While physical cross-sections provide a more thorough study of stratigraphy and chemical components, their use is limited to microscopic small areas. When contrast and resolution of OCT are appropriate to image the material under study, as is the case for the reconstructions here, OCT imaging has the benefit of non-invasive application. It is also time efficient and applicable to large areas [18]. In future research, we do aim to apply our approach to other research questions including the analysis of historic paintings.

\section{Conclusion}

In this work, we studied the morphology and mechanical properties of red glaze reconstructions containing ground glass with $\mathrm{OCT}$ and nanoindentation. 
Analysing the OCT data with a custom-developed analysis pipe-line yielded quantitative measurements for the size and spatial distribution of the added glass particles. The results showed a more homogeneous particle size distribution for the reconstruction (B4) in which the glass was additionally ground together with the oil/ pigment mixture. The mechanical properties of the different reconstructions were studied by nanoindentation measurements. We showed that the influence of the addition of glass increases the elastic and viscous moduli of the dried red glazes, and further investigated the differences resulting from the grinding process used to prepare the particles. The increase of the total surface area of the glass, with finer particles and a more homogeneous size distribution when the pigment, oil, and glass are ground together for an extra time, resulted in higher elastic and viscous moduli of the dried paint film.

The imaging and analysis approach described in this paper provides a tool for non-invasive investigation of ground glass in semi-transparent paint layers in both reconstructions and actual paintings. The results gathered on the mechanical behaviour and morphological properties are crucial steps for future research to further uncover the rationale behind the addition of glass to paints, and to understand the influence of the preparation process.

\section{Declarations}

\section{Competing interests}

The authors do not have any competing interest.

\section{Abbreviation \\ OCT: Optical Coherence Tomography.}

\section{Acknowledgements}

The authors thank Davide lannuzzi (Vrije Universiteit) for the nanoindentation research set up, Maurice Aalders (Biomedical Engineering and Physics department, Amsterdam UMC) for the OCT facilities and fruitful discussions, and Leah Wilk (Amsterdam UMC) for assistance with the data-analysis pipeline. The authors also thank the members of the Imaging, Identification and Interpretation of Glass in Paint project.

\section{Authors' contributions}

MA, MT and EH conceived the research; MA and MT designed the research EH oversaw the study, historical research and the reconstructions; MA and MT performed the experiments. Nanoindentation data-analysed by MT; OCT data-analysis pipeline and analysis by LF and MA; all the authors contributed to the manuscript preparation and writing, and approved the final version. All authors read and approved the final manuscript.

\section{Funding}

The research leading to the nanoindentation results has gratefully received funding from the Koninklijke De Heus/Rijksmuseum Fonds. The research is part of the Imaging, Identification and Interpretation of Glass in Paint project, funded by Netherlands Organisation for Scientific Research (NWO), under the umbrella of the Netherlands Institute for Art, Conservation and Science (NICAS). Partial funding from NSF PIRE (\#1743748): Computationally-Based Imaging of Structure in Materials (CUBISM) is gratefully acknowledged.

\section{Availability of data and materials}

The datasets used and/or analysed during the current study are available from the corresponding author on reasonable request.

\section{Author details}

${ }^{1}$ Conservation and Science Department, Rijksmuseum Ateliergebouw, Hobbemastraat 22, 1071 ZC Amsterdam, Netherlands. ${ }^{2}$ Biomedical Engineering and Physics, Amsterdam UMC, University of Amsterdam, Amsterdam, The Netherlands. ${ }^{3}$ Engineering Science and Applied Mathematics, Northwestern University, Chicago, II, USA. ${ }^{4}$ History of Art Department, University of Amsterdam, 1012 WX Amsterdam, Netherlands.

Received: 6 February 2021 Accepted: 29 April 2021

Published online: 04 June 2021

\section{References}

1. Dietz S. Malen mit Glas-Studien zur Maltechnik von Hans Holbein d. ̈. PhD thesis, Kölner Schriften zur Geistes- und Gesellschaftswissenschaftlichen Forschung, 2016.

2. Spring M. Colourless Powdered Glass as an Additive in Fifteenth-and Sixteenth-Century European Paintings. Natl Gall Tech Bull. 2012;33:4-26.

3. Seccaroni C, Moioli P, Borgia I, Brunetti B, Sgamellotti A. The painting technique of Pietro Vannucci, called il Perugino. In: Brunetti BG, Seccaroni C, Sgamellotti A, editors. Proc LabS Tech Work Quad di Kermes. Florence; 2004.

4. Spring M. Perugino's painting materials: analysis and context within sixteenth century easel paintings. In: Brunetti BG, Seccaroni C, Sgamellotti A, editors. The painting technique of Pietro Vannucci, called il Perugino. Perugia: Proc LabS Tech Work Quad di Kermes; 2004.

5. Spring M. Raphael's materials: Some new discoveries and their context within early sixteenth-century painting. In: Roy A, Spring M, editors. Raphael's painting technique: working practices before Rome. London: Proc EU-ARTECH Work Quad di Kermes; 2004.

6. Amadori ML, Poldi G, Barcelli S, Baraldi P, Berzioli M, Casoli A, et al. Lorenzo Lotto's painting materials: an integrated diagnostic approach. Spectrochim Acta A Mol Biomol Spectrosc England. 2016;164:110-22.

7. Dik J, Hermens E, Peschar R, Schenk H. Early production recipes for lead antimonate yellow in itallian art. Hoboken: Archaeometry. John Wiley \& Sons; 2005.

8. Melo HP, Cruz AJ, Valadas S, Cardoso AM, Candeias A. The use of glass particles and its consequences in late 16th century oil painting: a Portuguese case based on the analytical results and the technical treatises. J Cult Herit. 2019;43:261-70.

9. Lutzenberger $\mathrm{K}$, Stege $\mathrm{H}$, Tilenschi $\mathrm{C}$. A note on glass and silica in oil paintings from the 15th to the 17th century. J Cult Herit. 2010;11:365-72.

10. Bartl A, Krekel C, Lautenschlager M, Oltrogge D. Oltrogge, Der “Liber illuministarum" aus Kloster Tegernsee - Edition, Übersetzung und Kommentar der kunsttechnologischen Rezepte, Franz Steiner Verlag. Stuttgart; 2005.

11. Berger E. Quellen für Maltechnik während der Renaissance und der Folgezeit. München; 1901.

12. Smith $M$. The art of painting according to the theory and practise of the best Italian, French, and Germane masters. London; 1692

13. The Making and Knowing Project, Smith PH, Rosenkranz N, Uchacz TH, Taape T, Godbarge C, Pitman S, Boulboullé J, Klein J, Bilak D, Smith M, Catapano T, editors. Secrets of craft and nature in renaissance France. A Digital Critical Edition and English Translation of BnF Ms. Fr. 640. New York: The Making and Knowing Project; 2020. http://edition640.makin gandknowing.org.

14. Veliz Z. Artists'Techniques in Golden Age Spain. Six treatises in translation. Cambridge University Press. Cambridge;1986

15. Merrifield MP. Original Treatises, dating from the Xllth to XVIIIth Centuries on the Arts of Painting. London; 1849

16. Cheung CS, Spring M, Liang H. Ultra-high resolution Fourier domain optical coherence tomography for old master paintings. Opt Express. 2015;23:10145-57.

17. Targowski P, Iwanicka M. Optical coherence tomography: Its role in the non-invasive structural examination and conservation of cultural heritage objects—a review. Appl Phys A Mater Sci Process. 2012;106:265-77. 
18. Callewaert T, Guo J, Harteveld G, Vandivere A, Eisemann E, Dik J, et al. Multi-scale optical coherence tomography imaging and visualization of Vermeer's Girl with a Pearl Earring. Opt Express. 2020;28:26239-56.

19. van Loon A, Noble P, de Man D, Alfeld M, Callewaert T, Van der Snickt $\mathrm{G}$, et al. The role of smalt in complex pigment mixtures in Rembrandt's Homer 1663: combining MA-XRF imaging, microanalysis, paint reconstructions and OCT. Herit Sci. 2020;8:90.

20. Tiennot M, Paardekam E, lannuzzi D et al. Mapping the mechanical properties of paintings via nanoindentation: a new approach for cultural heritage studies. Sci Rep. 2020;10:7924. https://doi.org/10.1038/ s41598-020-64892-7.
21. SCHOTT Zemax catalog 2017-01-20b.

22. Berg S, Kutra D, Kroeger T, Straehle CN, Kausler BX, Haubold C, et al. ilastik: interactive machine learning for (bio)image analysis. Nat Methods. 2019;16:1226-32.

23. Callewaert T, Dik J, Kalkman J. Segmentation of thin corrugated layers in high-resolution OCT images. Opt Express. 2017;25:32816-28.

\section{Publisher's Note}

Springer Nature remains neutral with regard to jurisdictional claims in published maps and institutional affiliations.

\section{Submit your manuscript to a SpringerOpen ${ }^{\circ}$ journal and benefit from:}

- Convenient online submission

- Rigorous peer review

- Open access: articles freely available online

- High visibility within the field

- Retaining the copyright to your article

Submit your next manuscript at $\boldsymbol{\nabla}$ springeropen.com 